\title{
Comparison of the electromyographic activity of the tibialis anterior and gastrocnemius in stroke patients and healthy subjects during squat exercise
}

\author{
Dong-Kyu Lee, PT, MSc ${ }^{1)}$, Jun-Seok Kim, PT, BHSc ${ }^{2)}$, Tae-Hoon Kim, OT, PhD ${ }^{3)}$, \\ JAE-SEOP OH, PT, $\left.\mathrm{PhD}^{4}\right)^{*}$ \\ 1) Department of Rehabilitation Science, Graduate School, Inje University, Republic of Korea \\ 2) Department of Physical Therapy, Graduate School, Inje University, Republic of Korea \\ 3) Department of Occupational Therapy, Dongseo University, Republic of Korea \\ 4) Department of Physical Therapy, College of Biomedical Science and Engineering, Inje University: \\ 607 Obang-dong, Gimhae-si, Gyeongsangnam-do 621-749, Republic of Korea
}

\begin{abstract}
Purpose] The purpose of this study was to compare the EMG activity of the tibialis anterior (TA) and gastrocnemius (GCM) during the downward, maintenance, and upward phases of the squat exercise and during passive ankle dorsiflexion range of motion between stroke patients and healthy subjects. [Subjects] Fifteen hemiplegic ( 8 males, 7 females) and 15 healthy subjects ( 4 males, 11 females) volunteered for this study. [Methods] All subjects performed a double-leg squat exercise with the knee joint flexed to $30^{\circ}$. Surface electromyography (EMG) signals were recorded from the TA and GCM on the paretic or nondominant side. Passive ankle dorsiflexion range of motion (DF PROM) was measured using a goniometer in the knee-extended prone position. [Results] In the downward and maintenance phases, TA activity was significantly higher in stroke patients compared with healthy subjects. In the upward phase, GCM activity was significantly lower in stroke patients compared with healthy subjects. Ankle DF PROM was significantly lower in stroke patients compared with healthy subjects. [Conclusion] The observed EMG patterns should be taken into consideration to inform and enhance therapy for stroke patients. Key words: EMG, Squat exercise, Stroke
\end{abstract}

(This article was submitted Jun. 27, 2014, and was accepted Aug. 19, 2014)

\section{INTRODUCTION}

The majority of stroke patients experience muscle weakness $^{1)}$ and motor impairments, such as excessive contraction of muscles ${ }^{2)}$ and reduced EMG burst ${ }^{3}$. Poststroke physical capacity has been reported to be reduced by as much as $40 \%$ relative due to the physical capacity of healthy persons ${ }^{4}$, and this is due to loss of muscle strength and excessive activation, which consequently lead to functional deficits.

Functional recovery of the lower limbs is an important aspect of rehabilitation following stroke. $\mathrm{Ng}$ et al. reported that impaired ankle dorsiflexion strength is a crucial component in determining the Time Up and Go performance of individuals with spastic hemiplegia ${ }^{5)}$. EMG overactivity of the tibialis anterior (TA) leads to forefoot varus during the swing phase ${ }^{6}$. The ankle plantar flexors are known to generate the majority of the power required for forward gait progression ${ }^{7}$. Cooper et al. reported a strong relationship between ankle plantar flexor weakness (especially the

*Corresponding author. Jae-Seop Oh (E-mail: ysrehab@inje. ac.kr)

(C2015 The Society of Physical Therapy Science. Published by IPEC Inc. This is an open-access article distributed under the terms of the Creative Commons Attribution Non-Commercial No Derivatives (by-ncnd) License $<$ http://creativecommons.org/licenses/by-nc-nd/3.0/>. gastrocnemius; GCM) and knee hyperextension during the mid-stance phase ${ }^{8)}$.

Clinically, the squat exercise is used to strengthen the lower limbs following stroke ${ }^{9}$. The squat can be used in neurorehabilitation to safely retrain motor control during whole-body movements; it also involves bilateral movements ${ }^{10}$. Weight-bearing exercises, especially squats, are effective in improving the function of lower extremity muscles because they involve both downward and upward movements of the body, with flexion and extension of the hip, knee, and ankle occurring simultaneously ${ }^{11)}$. The squat has been studied extensively in healthy individuals with specific reference to the TA and GCM, but not in stroke patients.

The primary purpose of this study was to compare the EMG activity in the TA and GCM during the downward, maintenance, and upward phases of the squat exercise in stroke patients compared with healthy subjects. A secondary objective was to investigate potential group differences in passive ankle dorsiflexion range of motion (DF ROM).

\section{SUBJECTS AND METHODS}

Fifteen hemiplegic patients ( 8 males, 7 females) were recruited from Dong-eui Medical Center, Busan, Republic of Korea. Their mean age was $59.8 \pm 8.4$ years, and their 
mean height and weight were $165.1 \pm 7.9 \mathrm{~cm}$ and $64.1 \pm$ $7.1 \mathrm{~kg}$, respectively. Four functionality scales were used to assure homogeneity and minimize selection bias: subjects were eligible if they had a Berg Balance Scale (BBS) score between 35-45, a Korean-Modified Barthel Index (K-MBI) score between 60-85, a Modified Ashworth Scale (MAS), score between 1-2, and a Mini-Mental Status ExaminationKorean version (MMSE-K) score above 24. The mean BBS, K-MBI, MAS, and MMSE-K scores of the patients were $41.7 \pm 3.4,80.4 \pm 4.0,1.5 \pm 0.5$, and $29.2 \pm 1.4$, respectively. Hemiplegia following their first unilateral stroke, which was required to have occurred more than 6 months previously, represented a further inclusion criterion. The mean poststroke interval of the patients was $10.6 \pm 7.5$ months. Fifteen healthy subjects (4 males, 11 females) were recruited from the community. Their mean age was $55.0 \pm 9.9$ years, and their mean height and weight were $162.7 \pm 4.1 \mathrm{~cm}$ and $60.2 \pm 6.3 \mathrm{~kg}$, respectively. Healthy subjects were excluded if they reported any current neurological or musculoskeletal pain. Subjects with disabilities that might impair task performance of the squat exercise with knee flexion of $30^{\circ}$ or with comprehension deficits were excluded. Prior to participation, all subjects read and signed an informed consent form approved by the Inje University Ethics Committee for Human Investigations.

To acquire EMG signals, surface EMG data were recorded using a Trigno wireless EMG system (Delsys, Inc., Boston, MA, USA). EMG data were collected from the TA and GCM on the paretic or nondominant side (preferred leg for kicking a ball). Electrodes were situated as follows: for the TA, one quarter to one third of the distance between the knee and the ankle, and for the GCM, immediately distal from the knee and $2 \mathrm{~cm}$ medial to the midline ${ }^{12)}$. Data analysis was performed using the EMGworks software package (ver. 4.0; Delsys). The sampling rate for the EMG signal was set at $1,000 \mathrm{~Hz}$; the band-pass filter was set between 20-450 Hz. Raw data from the TA and GCM muscles were transformed into root mean square (RMS) data. The mean RMS of the reference voluntary contraction (RVC) was calculated for each muscle when subjects were in a comfortable standing position for normalization of the data ${ }^{13)}$. The EMG data were collected during a holding time of $5 \mathrm{~s}$ in a standing position. For the data analysis, we used $3 \mathrm{~s}$ of the $5 \mathrm{~s}$ of EMG data, excluding the initial $1 \mathrm{~s}$ and final 1 s. All EMG data are expressed as percentages of the RVC (\%RVC).

Prior to testing, all subjects were appraised of the methods involved and practiced to gain familiarity with the procedure. Each subject was instructed to stand with the hips and knees fully extended and the feet positioned shoulderwidth apart with the toes pointing directly forward. A goniometer was used to determine that the knee joint range was at $30^{\circ}$; a target bar was then placed on the patella to provide feedback. Subjects were requested to stand, starting from a position at which the knee joint was flexed to $30^{\circ}$, for $3 \mathrm{~s}$ until they made contact with the target bar. Following maintenance of $30^{\circ}$ knee flexion for $3 \mathrm{~s}$, the subjects then returned to the starting position for a further $3 \mathrm{~s}$. Phase timing of the squats was controlled using a series of beeps,
Table 1. EMG activity (\%RVC) of the tibialis anterior and gastrocnemius during squat

\begin{tabular}{lccc}
\hline & & Stroke $(\mathrm{n}=15)$ & Healthy $(\mathrm{n}=15)$ \\
\hline Downward & TA & $330.8 \pm 181.9^{*}$ & $207.8 \pm 74.4$ \\
& GCM & $132.3 \pm 51.3$ & $168.7 \pm 112.3$ \\
Maintenance & TA & $346.7 \pm 229.7^{*}$ & $199.8 \pm 68.7$ \\
& GCM & $138.8 \pm 54.6$ & $155.0 \pm 94.9$ \\
Upward & TA & $292.5 \pm 190.1$ & $200.6 \pm 73.3$ \\
& GCM & $179.2 \pm 63.8^{*}$ & $300.3 \pm 204.9$ \\
\hline
\end{tabular}

Data are expressed as the mean \pm SD. TA: tibialis anterior, GCM: gastrocnemius. ${ }^{*} \mathrm{p}<0.05$

generated at 1-s intervals, by a simulated metronome. EMG activity was continuously recorded during the downward, maintenance, and upward phases of the squat exercise. Following completion of the squat, DF PROM was measured using a goniometer by a physical therapist with 6 years of experience pertaining to the knee-extended prone position.

Test trials were repeated three times, with a 1-min rest between trials. The mean values of the normalized EMG activity for the TA and GCM were used in the analysis of the downward, maintenance, and upward phases of the squat. For data analysis purposes, we used 2 of the $3 \mathrm{~s}$ of the EMG data recorded for the TA and GCM muscles, excluding the initial $0.5 \mathrm{~s}$ and final $0.5 \mathrm{~s}$.

The SPSS software package (ver. 18.0; SPSS Inc., Chicago, IL, USA) was used to compare the two groups' EMG activity in the tested muscles during each squat phase together with their DF PROM. Group differences were assessed using independent t-tests, and significance was defined as $\mathrm{p}$ $<0.05$.

\section{RESULTS}

In the downward and maintenance phases of the squat exercise, TA activity was significantly higher in the stroke patients compared with the healthy subjects $(\mathrm{p}<0.05)$. The $\%$ RVC values of the TA during the downward phase of the squat in the stroke patients and healthy subjects, respectively, were $330.8 \pm 181.9$ and $207.8 \pm 74.4$. In the maintenance phase, the \%RVC values of the TA in the stroke patients and healthy subjects, respectively, were $346.7 \pm 229.7$ and $199.8 \pm 68.7$. In the upward phase, the GCM activity was significantly lower in the stroke patients compared with the healthy subjects $(\mathrm{p}<0.05)$. The \%RVC values of the GCM in the stroke patients and healthy subjects, respectively, were $179.2 \pm 63.8$ and $300.3 \pm 204.9$ (Table 1).

Ankle DF PROM was significantly lower in the stroke patients compared with the healthy subjects $(\mathrm{p}<0.05)$. The angles of ankle DF PROM in the stroke patients and healthy subjects were $10.4 \pm 1.8$ and $20.2 \pm 2.1$, respectively (Table 2).

\section{DISCUSSION}

In this study, we compared the EMG activity of the TA and GCM in stroke patients and healthy subjects during the 
Table 2. Differences in passive dorsiflexion range of motion between the two groups $\left(^{\circ}\right)$

\begin{tabular}{ccc}
\hline & Stroke $(n=15)$ & Healthy $(n=15)$ \\
\hline DF ROM & $10.4 \pm 1.8^{*}$ & $20.2 \pm 2.1$ \\
\hline
\end{tabular}

Data are expressed as the mean $\pm \mathrm{SD}$.

DF ROM: differences in passive dorsiflexion range of motion between the two groups $\left(^{\circ}\right)$. $* \mathrm{p}<0.05$

squat exercise. Stroke patients had greater activity in the TA compared with healthy subjects during the downward and maintenance phases of the squat, but the GCM activity was lower in stroke patients compared with healthy subjects.

A contraction in the TA initiates the downward phase of the squat exercise, thereby assisting dorsiflexion ${ }^{11)}$. Gray et al. reported that deficits in the paretic TA were observed during the initiation and termination phases of the squat in stroke patients compared with healthy subjects ${ }^{10)}$. However, our study indicated that stroke patients had greater activity in the TA compared with healthy subjects in the downward and maintenance phases. In the study by Gray et al., subjects performed a squat with approximately $30^{\circ}$ knee flexion in which they were instructed to perform the downward movement as quickly as possible ${ }^{10)}$; however, our study required subjects to stand, starting from a position in which the knee joint was flexed to $30^{\circ}$, for $3 \mathrm{~s}$ until they made contact with the target bar. Thus, the decreased speed when performing the squats may have increased TA activity in stroke patients because they have a limited capacity for ankle dorsiflexion. Furthermore, limited dorsiflexion during weight-bearing tasks encourages increased subtalar joint pronation and tibial internal rotation ${ }^{14)}$. The results of our study indicated that ankle DF PROM was significantly lower in stroke patients compared with healthy subjects. This may explain the overactivity in the paretic TA of the stroke patients during the downward and maintenance phases of the squats, because the TA may contribute to inversion of the foot accompanied by a heel lift. According to Day et al., in tasks of increasing postural demand, where greater TA activation is required ${ }^{15)}$. Therefore, impairment of balance in hemiplegic patients may be influence TA muscle activity to maintain posture.

The GCM, the biarticular knee flexor, was actively recruited during the upward phase of the squats and, similarly to the hamstrings, contracted eccentrically ${ }^{11)}$. Previous research has demonstrated that the nature of contraction influences the degree of deficit observable in the paretic muscles, with less impairment during eccentric contractions and greater deficits during concentric contractions ${ }^{16,17)}$. The present study indicated that eccentric extension is impaired, as evidenced by the decreased GCM activity. This may engender a compensatory strategy of overuse of the non-paretic leg to overcome the weak activation of muscles in the paretic leg. It is also suggested that shortening of the ankle plantar flexors might limit the dorsiflexion range, which could impair activation of the GCM.
In conclusion, we demonstrated that paretic leg muscular activity in stroke patients differs from that of healthy subjects during the downward, maintenance, and upward phases of the squat exercise, with overactivity of the TA and decreased muscle activity of the GCM indicative of impairment. In addition, this study provided information pertaining to abnormalities in muscle activation following stroke, which could inform future rehabilitation programs. Increasing ankle DF PROM, without restricting ankle movement, could be used as a treatment to improve the performance of the squat exercise, given that quantification of motor deficits is essential to therapeutic approaches for stroke patients. Future investigations should aim to evaluate the effects of stretching on muscle activation in the context of ankle dorsiflexion.

\section{REFERENCES}

1) Patten C, Lexell J, Brown HE: Weakness and strength training in persons with poststroke hemiplegia: rationale, method, and efficacy. J Rehabil Res Dev, 2004, 41: 293-312. [Medline] [CrossRef]

2) Dewald JP, Pope PS, Given JD, et al.: Abnormal muscle coactivation patterns during isometric torque generation at the elbow and shoulder in hemiparetic subjects. Brain, 1995, 118: 495-510. [Medline] [CrossRef]

3) Garland SJ, Willems DA, Ivanova TD, et al.: Recovery of standing balance and functional mobility after stroke. Arch Phys Med Rehabil, 2003, 84: 1753-1759. [Medline] [CrossRef]

4) Macko RF, Benvenuti F, Stanhope $S$, et al.: Adaptive physical activity improves mobility function and quality of life in chronic hemiparesis. J Rehabil Res Dev, 2008, 45: 323-328. [Medline] [CrossRef]

5) Ng SS, Hui-Chan CW: Ankle dorsiflexor, not plantarflexor strength, predicts the functional mobility of people with spastic hemiplegia. J Rehabil Med, 2013, 45: 541-545. [Medline] [CrossRef]

6) Perry J: The use of gait analysis for surgical recommendations in traumatic brain injury. J Head Trauma Rehabil, 1999, 14: 116-135. [Medline] [CrossRef]

7) Winter DA: Energy generation and absorption at the ankle and knee during fast, natural, and slow cadences. Clin Orthop Relat Res, 1983, (175): 147-154. [Medline]

8) Cooper A, Alghamdi GA, Alghamdi MA, et al.: The relationship of lower limb muscle strength and knee joint hyperextension during the stance phase of gait in hemiparetic stroke patients. Physiother Res Int, 2012, 17: 150-156. [Medline] [CrossRef]

9) Pang MY, Eng JJ: Determinants of improvement in walking capacity among individuals with chronic stroke following a multi-dimensional exercise program. J Rehabil Med, 2008, 40: 284-290. [Medline] [CrossRef]

10) Gray VL, Ivanova TD, Garland SJ: Control of fast squatting movements after stroke. Clin Neurophysiol, 2012, 123: 344-350. [Medline] [CrossRef]

11) Robertson DG, Wilson JM, St Pierre TA: Lower extremity muscle functions during full squats. J Appl Biomech, 2008, 24: 333-339. [Medline]

12) Cram JR, Kasman GS, Holtz J: Introduction to surface electromyography. Gaithersburg: Aspen, 1998.

13) Lee MH, Shim JM, Park MC, et al.: A study of the stance limb during the crossing of obstacles of different heights by hemiplegic stroke patients. J Phys Ther Sci, 2010, 22: 317-321. [CrossRef]

14) Macrum E, Bell DR, Boling M, et al.: Effect of limiting ankle-dorsiflexion range of motion on lower extremity kinematics and muscle-activation patterns during a squat. J Sport Rehabil, 2012, 21: 144-150. [Medline]

15) Day JT, Lichtwark GA, Cresswell AG: Tibialis anterior muscle fascicle dynamics adequately represent postural sway during standing balance. J Appl Physiol 1985, 2013, 115: 1742-1750. [Medline] [CrossRef]

16) Lum PS, Patten C, Kothari D, et al.: Effects of velocity on maximal torque production in poststroke hemiparesis. Muscle Nerve, 2004, 30: 732-742. [Medline] [CrossRef]

17) Clark DJ, Condliffe EG, Patten C: Activation impairment alters muscle torque-velocity in the knee extensors of persons with post-stroke hemiparesis. Clin Neurophysiol, 2006, 117: 2328-2337. [Medline] [CrossRef] 